\title{
The Impact of Google Hacking on Identity and Application Fraud
}

\author{
Amany Abdelhalim, Issa Traore \\ Department of Electrical and Computer Engineering \\ University of Victoria, P.O.Box 3055 STN CSC, \\ Victoria, B.C., V8W 3P6, Canada \\ Email: \{amany, itraore\}@ece.uvic.ca
}

\begin{abstract}
In the last several years, identity theft has been on the rise. The Internet represents an appealing place for fraudsters to collect a host of personal and financial data related to many innocent users. Using the collected data they can impersonate the users and commit different fraudulent activities including application fraud. Mining Internet data for fraudulent purposes is commonly referred to as (black hat) Google hacking. We discuss in this paper the impact of Google hacking on identity fraud, with an emphasis on fraudulent applications for identity certificates such as credit cards, passports, and so on. The discussion is based on the results of an experiment performed over the Internet by conducting some (white hat) Google hacking and collecting sensitive identity information for living as well as dead persons. We also outline the architecture of a security tool for detecting application fraud that is currently under development.
\end{abstract}

\section{INTRODUCTION}

In the last several years, identity theft has been on the rise and become a key concern for most government and private institutions. Fraudsters commit fraudulent activities using either stolen or fake identities. In this context, the Internet represents an appealing place for fraudsters to collect a host of personal and financial data related to many innocent users. Powerful search engines such as Google are used by hackers in malicious ways to collect identity information over the web. Such process is referred to as black hat Google hacking.

Identity fraud occurs when a criminal impersonates another individual by taking on that person's identity for whatever reason [1], [2]. In the literature, identity frauds are categorized into two classes, namely application fraud and behavioral fraud. Application fraud corresponds to the process of applying and obtaining an identity certificate (e.g., passport, credit card etc.) using someone else's identity.

Behavioral fraud occurs when fraudulent transactions are performed by individuals who have already stolen identity information. Most of the previous works in the area of identity theft prevention and detection have focused, so far, on credit card fraud from the behavioral point of view.

These typically involve the detection of suspicious credit card transaction patterns that seem to be related to someone else other than the actual card owner. In this work, we are interested primarily in studying the effectiveness of using identity information that is available online in detecting fraudulent applications. We aim for targeting a broader range of frauds beside credit card frauds. The ultimate goal of our research is to develop an automated framework for detecting application fraud based on identity information that is available online.

In this paper, we present and discuss the results of an exploratory experiment of a manual search based on Google that explores the existence of identity information over the web not targeting a specific individual. In the general context of our research, this study is important as it allowed us to have a sense of the types of pieces of information out there that could fall into the wrong hands, and to identify and develop appropriate mechanisms to detect fraudulent activities.

\section{IDENTITY INFORMATION SOURCES}

The main tools used in online identity theft are search engines. Search engines index a huge number of Web pages and other resources that sometimes expose security weaknesses and confidential data. Millions of Internet users $\log$ in daily to conduct various activities such as job searching, filling applications for various services, conducting financial transactions and so on. Many users key in their names, addresses, home and work telephones, fax, electronic mail addresses, or credit card numbers without any hesitation. Many such pieces of identity information could be found by thieves on the internet either for free or with little money. A thief can submit to a search engine a few pieces of information that he already knows and combine them in different ways for obtaining more information. As a result he could gather a lot of information such as tax records, social security numbers, credit card information and others [3].

Google offers particular appeal over other search engines because of its effectiveness in locating specific information. Google has become one of the main tools for hackers because it has many powerful search options. Google is also useful in finding credit card and social security data because it gives you an ability to search for ranges of numbers [4].

There are many web sites providing lists of financial information that include hundreds of card holders' personal information along with their credit card data. Governments also produce many files which are available on the net, such as divorce-court records, that could include banking information [4]. 
Online resumes posted by individual job seekers or by businesses for their employees, often contain personal information such as social security numbers, dates of birth, home/office addresses, phone numbers, and sometimes family information [5].

\section{WHITE HAT GOOGLE HACKING}

Identity thieves have many ways, legal or illegal, for obtaining much of the necessary personal information for perpetrating identity fraud by simply using the public information sources available online. In order to achieve an understanding of the impact of this situation and identify an appropriate strategy to mitigate such impact, we conducted some white hat Google hacking experiments over several weeks. We were able to collect social security numbers, dates of birth and addresses for many people. We found this kind of information in online resumes, loan statements, tax payments, wanted criminals' lists, companies' advertisements for delivery services, posted petitions for dissolution of marriage, and posted court complaints. In order to search for such information, we used different words that express identity information, such as the social security number, and searched in different types of documents.

TABLE 1. EXAMPLES OF SEARCH QUERIES FOR RETRIEVING SSN INFORMATION FOR ALIVE PEOPLE.

\begin{tabular}{|c|}
\hline Social Security Queries \\
\hline Ssn \\
\hline social security number \\
\hline social security number OR cv OR resume OR curriculum Vitae \\
\hline ssn OR cv OR resume OR curriculum Vitae \\
\hline ssn\# OR ssnum OR ssno \\
\hline
\end{tabular}

By manually searching for identity information using Google in a short time period, we were able to collect sensitive identity information for living as well as for dead persons. Tables 1-3 depict examples of keywords used in the queries used to search for social security and credit card information. Google also made it easier for us to find credit card numbers by searching for ranges of numbers. We were able to collect social security numbers, as well as credit card numbers along with their expiry dates and security numbers from purchases, payrolls, donations, and hotel reservations posted online. Table 4-8 summarizes the information collected.

TABLE 2. EXAMPLES OF SEARCH QUERIES FOR RETRIEVING DECEASED SSN INFORMATION.

\begin{tabular}{|c|}
\hline Query \\
\hline ssn AND died \\
\hline ssn AND death \\
\hline social security number AND died \\
\hline
\end{tabular}

\begin{tabular}{|c|}
\hline social security number AND death \\
\hline death records OR death certificates \\
\hline genealogy site \\
\hline
\end{tabular}

TABLE 3. EXAMPLES OF SEARCH QUERIES FOR RETRIEVING CREDIT CARD INFORMATION.

\begin{tabular}{|c|}
\hline Credit Card Queries \\
\hline credit card number OR creditno OR creditnum OR ccnum OR ccno \\
OR cc\# filetype:xls \\
\hline credit card type OR card type OR Ctype filetype:xls \\
\hline credit expire date OR expire date OR e-date OR expdate filetype:xls \\
\hline visa OR master card OR MC OR amex filetype:xls \\
\hline
\end{tabular}

We were able also to collect a lot of social security information for deceased people from genealogy sites and posted death certificates in a short period of time which are summarized in table 4. The Social Security Death Index alone contains more than 78 million records, each containing a social security number of a dead person and it is freely accessible online by anyone.

TABLE 4. SUMMARY OF THE RECORDS COLLECTED FOR THE SSN INFORMATION FOR DECEASED PEOPLE.

\begin{tabular}{|c|c|c|}
\hline \multicolumn{3}{|c|}{1160 Social Security records including } \\
\hline Name & Ssn & Date of Birth \\
\hline 1160 & 1160 & 1133 \\
\hline
\end{tabular}

Tables 5-8 list the different pieces of identity information retrieved for living people and the corresponding quantities. These include social security information, passport information, credit card information, and health information, RESPECTIVELY.

TABLE 5. SUMMARY OF THE SSN RECORDS COLLECTED FOR ALIVE PEOPLE.

\begin{tabular}{|c|c|c|c|c|}
\hline \multicolumn{5}{|c|}{155 Social Security records including } \\
\hline Name & Ssn & $\begin{array}{c}\text { Date of } \\
\text { Birth }\end{array}$ & Address & $\begin{array}{c}\text { Telephone } \\
\text { Number }\end{array}$ \\
\hline 155 & 155 & 57 & 46 & 36 \\
\hline
\end{tabular}

TABLE 6. SUMMARY OF THE PASSPORT INFORMATION COLLECTED.

\begin{tabular}{|c|c|c|}
\hline \multicolumn{3}{|c|}{ 2 Passport records including } \\
\hline Passport Number & $\begin{array}{c}\text { Passport Issuing } \\
\text { Date }\end{array}$ & $\begin{array}{c}\text { Passport Expiry } \\
\text { Date }\end{array}$ \\
\hline 2 & 2 & 2 \\
\hline
\end{tabular}

TABLE 7. SUMMARY OF THE CREDIT CARD INFORMATION COLLECTED.

\begin{tabular}{|c|c|c|c|}
\hline \multicolumn{5}{|c|}{28 credit card records including } \\
\hline $\begin{array}{c}\text { Credit Card } \\
\text { number }\end{array}$ & Card Type & $\begin{array}{c}\text { Card Expiry } \\
\text { Date }\end{array}$ & $\begin{array}{c}\text { Card } \\
\text { Verification } \\
\text { Number }\end{array}$ \\
\hline 28 & 26 & 19 & 2 \\
\hline
\end{tabular}


TABLE 8. SUMMARY OF THE HEALTH INFORMATION COLLECTED.

\begin{tabular}{|c|c|}
\hline \multicolumn{2}{|c|}{1 health record including } \\
\hline Name & Medicare Card Number \\
\hline 1 & 1 \\
\hline
\end{tabular}

The huge number and diversity of the information gathered in this short time span illustrates how dangerous black hat Google hacking can be for the privacy of many innocent and unsuspecting users. Besides the confirmation of this fact, this exploratory study has allowed us to establish a database of online identity information, which will serve as basis for the validation of our identity fraud detection framework.

\section{APPLICATION FRAUD DETECTION}

\section{A. State of the Art}

In [6], Bolton et al. categorize identity fraud into two classes, namely application fraud and behavioral fraud. They define behavioral fraud as fraudulent transactions made by thieves who have already stolen necessary identity information. They define application fraud as the process of obtaining an identity certificate from a certificate issuer using someone else's identity. Application fraud is the main focus of the research described in this paper.

In [7], Wheeler et al. proposed an application that deploys case-based reasoning to solve the problem of detecting application fraud. Their goal is to reduce the fraud investigations by combining the diagnosis of multiple algorithms. Their case based reasoning framework consists of two decision making components; the retrieval component which utilizes two algorithms the weight matrix and the nearest neighbor algorithms. The second component is the retrieval component which utilizes a set of multiple algorithms (probabilistic curve selection, best match algorithm, negative selection algorithm, density selection algorithm, and default goal) that are used to analyze the data retrieved from the first component.

The results of testing the system showed that the performance of each algorithm differs depending on the fraud case presented, in other words the nature of the fraud detection case.

In [8], Phua et al. proposed a technique for detecting application fraud called "the approximate pair wise score" that generates numeric scores that describes the application suspicion degree. In their technique they classify a new application as belonging to one of three lists, either a black list, white list or an anomalous list. They do the classification by finding a match between the new application and an application in one of the lists. Both the new application and the application that is going to be compared to it are represented by an attribute vector. The suspicious score is the summation of each pair-wise attribute which is described be either exact matching or fuzzy matching. They also assign weights to the attributes depending on their nature. Their proposed technique is similar to the weight matrix algorithm deployed in [7]. The difference between the weight matrix technique in [7] and the approximate pair wise score technique in [8], is that in [8] in addition to basing their calculation of suspicion scores on matching attributes, they take into consideration the time and space difference between the matching applications. The data that they used for testing their algorithm is synthetic data created by a data generator. They state that the generator is not capable of creating normal communities yet.

The limitation of the weight matrix algorithm and the approximate pair wise score is that they assume that the person who will submit a fraudulent application will always be using instances of information that he already used before in subsequent fraudulent applications. This means that at the beginning all new fraudulent application cases will go undetected since there will be no similar fraud application to match against.

In [9], Burns et al. presented a discussion on managing fraud in the credit card industry. They described different fraudulent activities that are observed in the industry. One of these activities which we concern about is the application fraud. They divided application fraud into two forms "familiar" and "unfamiliar". Familiar application fraud occurs when someone close to the victim such as a friend or roommate fills out a credit card application that is sent to the victim and uses it when the card is received. The unfamiliar application fraud happens when an unknown person to the victim does the same act. They also outlined some tactics that card issuers follow in the process of screening a credit application. Card issuers use multiple data sources to confirm the information presented on an application. They also check with the information received from credit bureaus. They also calculate the distance between the phone number and address presented in the application to determine if they originate from the same area code or not. Applications which are submitted from areas where a lot of fraud cases appeared before are also reviewed thoroughly.

In [10], Estevez et al proposed a system that prevents subscription fraud in fixed telecommunications. The system consists of two main modules, a classification module and a prediction module, which is of interest in our research. The prediction module's goal is to be able to detect fraudulent customers when they attempt to subscribe to a fixed telecommunication service. In investigating the application for fraud, the prediction module crosses the information available in the new application with information available in the account database. This is done to try to find other lines installed at the same address of the new application where there owners were flagged as fraudulent customers. Another source of information that is used is a public database that lists situations of insolvency mostly related to banks and department stores. The prediction module consists of a 
multi-layer feed-forward neural network. The output units indicate one of two decisions for an application; either fraudulent or legitimate. A data set of subscription examples were labeled and split into the following three sets: a training set, a validation set, and a testing set. From their experiments they outlined that the prediction module was able to identify only $56.2 \%$ of the true fraudster cases while screening $3.5 \%$ of all the subscribers in the testing set.

In [11], [12], Sweeney proposed the Identity Angel project. The goal of the Identity Angel project is to locate online resumes that contain sufficient information for a criminal to apply online for a new credit card in the name of the resume owner. The program sends an email to the owner of the resume, encouraging him to remove such sensitive information.

\section{B. Architecture for an Application Fraud Detector}

Application for an identity certificate involves a collection of identity information that can be checked by collecting data online and structuring relevant identity information. By combining and analyzing the information provided by the applicant and the information collected online, we can detect and report possible identity fraud.

The profiles derived from the collected identity information can be used to detect identity fraud. We analyze the coherence of the profiles by checking the consistency of the identity information involved. For instance, one should expect that two different credit cards owned by the same individual will have the same mother's maiden name and address, or that a social security card and a credit card owned by the same individual will bear the same address.

The identity information retrieved for a particular individual consists of a collection of identity information patterns. Each pattern may consist of a sequence of identity information pieces such as address, birth date, credit card number, mother maiden name, social security number etc.

Identity fraud can be detected by screening a particular identity claim. An identity claim occurs when an individual declares a specific identity, for instance, on an official document such as a passport application, or a business document like a credit card application. The tool will extract the identity information for the individual that could be found online and from other available sources e.g. credit bureaus and check the results against the identity claim, reporting any discrepancy as an anomaly. This may either lead to a rejection of the application or to further investigations with some follow-up questions. The kinds of discrepancies that could be detected include, for instance, identity claims made in the name of dead people, or claims involving inconsistent addresses, dates of birth, professions and so on. Further checks may be conducted on the claim itself in order to detect some inconsistencies. For instance, a claim containing a telephone number that is inconsistent with the current address, or a social security number that is retired, would raise some flags.
Fig 1 depicts the general architecture of the tool, which consists of an identity information retrieval engine that uses the Google API, and feeds targeted search results into an identity fraud detector. The identity fraud detector combines and analyzes, using heuristics, the search results with background identity information fetched from the application file (e.g., credit card application form and credit bureau report) under scrutiny.

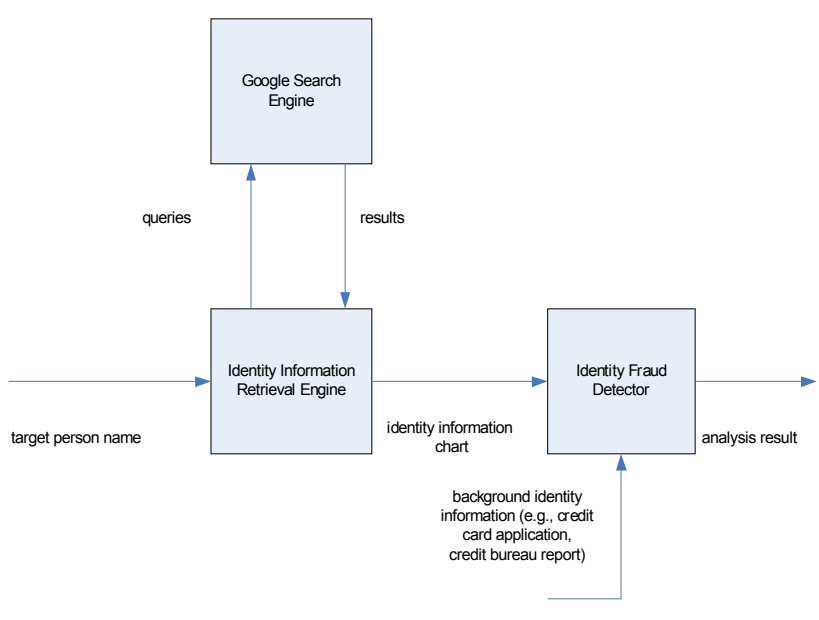

Fig 1. Tool Architecture.

\section{CONCLUSIONS}

The last several years, identity theft has been one of the fastest growing crimes. Unfortunately, the Internet has been facilitating this phenomenon since it represents a tremendous open repository for sensitive identity information available for those who know how to find them, including fraudsters. The long term goal of this research is to develop a security tool for finding identity information over the web that could be used for fraudulent applications for identity certificates. In this regard the results of the exploratory study presented in this paper are insightful and essential in developing appropriate mechanisms for fraud detection based on online information. In our future work we will develop the corresponding algorithms and mechanisms, and implement and evaluate our security tool.

\section{REFERENCES}

[1] Crown,"Identity fraud: a study", Economic and Domestic Secretariat Cabinet Office, July 2002, www.homeoffice.gov.uk/docs/id fraudreport.PDF.

[2] WenJie Wang; Yufei Yuan; Archer, N,"A contextual framework for combating identity theft", Security \& Privacy Magazine, IEEE Volume 4, March-April 2006 pp. $30-38$.

[3] Elizabeth A. Evans, Carolyn M. Kotlas, Donna W. Bailey, Abe J. Crystal, Terri Buckner. "It's eleven o'clock: do you know where your identity is?", Proceedings of the 32nd annual ACM SIGUCCS conference on User services, ACM Press, 2004. 
[4] Robert Lemos, "Google queries provide stolen credit cards", 2004, News.com.

http://news.com.com/Google+queries+provide+stolen+credit+cards/2100-

1029_3-5295661.html.

[5] Johnny Long, "Google hacking for penetration testers", book, 1st edition, Syngress, 2001.

[6] Richard J. Bolton and David J. Hand. "Unsupervised profiling methods for fraud detection", Credit Scoring and Credit Control Volume 2, Edinburgh, UK, 2001.

[7] Richard Wheeler, Stuart Aitken, "Multiple algorithms for fraud detection", Knowledge-Based Systems, 2000, pp. 93-99.

[8] Phua C, Gayler R, Lee V and Smith K (2006) "On the communal analysis suspicion scoring for identity crime in streaming credit applications", European Journal of Operational Research, submitted.

[9] Peter Burns, Anne Stanley, 2002. "Fraud management in the credit card industry," Payment Cards Center Discussion Paper 02-05, Federal Reserve Bank of Philadelphia.

[10] Pablo A. Estevez, Claudio M. Held and Claudio A. Perez "Subscription fraud prevention in telecommunications using fuzzy rules and neural networks", , Expert Systems with Applications Volume 31, Issue 2, August 2006, pp. 337-344.

[11] L. Sweeney, "Ai technologies to defeat identity theft vulnerabilities", AAAI Spring Symposium on AI Technologies for Homeland Security, 2005.

[12] L. Sweeney, "Protecting job seekers from identity theft", IEEE Internet Computing, 2006. 